\title{
Non-linear Jordan triple automorphisms of sets of self-adjoint matrices and operators
}

\author{
by \\ Lajos Molnár (Debrecen)
}

\begin{abstract}
We consider the so-called Jordan triple automorphisms of some important sets of self-adjoint operators without the assumption of linearity. These transformations are bijective maps which satisfy the equality

$$
\phi(A B A)=\phi(A) \phi(B) \phi(A)
$$

on their domains. We determine the general forms of these maps (under the assumption of continuity) on the sets of all invertible positive operators, of all positive operators, and of all invertible self-adjoint operators.
\end{abstract}

1. Introduction and statements of the results. Bijective multiplicative maps on rings and algebras have been studied by many authors. One motivation for such investigations comes from the problem of the automatic additivity of semigroup isomorphisms between rings. This question was first studied by Martindale in [12] where he obtained the surprising result that every semigroup isomorphism from a prime ring containing a non-trivial idempotent onto an arbitrary ring is necessarily additive. The same problem for operator algebras was treated, for example, in the papers $[3,8,19]$ of Hakeda, Lu and Šemrl. Similar questions concerning maps on operator algebras which are multiplicative with respect to other products such as the Jordan product $A B+B A$ or the Jordan triple product $A B A$ were investigated in the papers $[4-7,9-11,14,15]$ by Hakeda and Saitô, $\mathrm{Li}$ and Jing, Ling and $\mathrm{Lu}$, and the present author. These products play an important role in certain parts of ring theory and also in the mathematical foundations of quantum mechanics.

In the present paper we continue the above mentioned investigations. Namely, we consider the so-called Jordan triple automorphisms of certain

2000 Mathematics Subject Classification: Primary 47B49.

Key words and phrases: Jordan triple automorphism, self-adjoint operators, positive operators.

The author was supported by the Hungarian National Foundation for Scientific Research (OTKA), Grant No. T043080, T046203. 
important subsets of operator algebras (not of the "full" algebra). These transformations are bijective maps which satisfy the equality

$$
\phi(A B A)=\phi(A) \phi(B) \phi(A)
$$

on their domains. Below we determine the general forms of these automorphisms (under the assumption of continuity) on the sets of all invertible positive operators, of all positive operators, and of all invertible self-adjoint operators. (Observe that the sets in question are all closed under the Jordan triple product, but this is not the case for the Jordan product.) The same problem concerning the set of all self-adjoint operators was already solved in $[13]$.

We remark that, as one can see from the proofs, the present problem is harder than the ones concerning "full" operator algebras which were considered before in the literature. The reason is that the domains of our transformations are rather small, they contain only "nice" operators. It turns out that in the present situation our maps are usually non-linear.

We now turn to the formulations of our results. First let us fix the notation. In what follows $H$ denotes a complex separable Hilbert space with $\operatorname{dim} H \geq 3$. The algebra of all bounded linear operators on $H$ is denoted by $B(H)$. The symbols $B_{\mathrm{s}}(H), B_{\mathrm{s}}^{-1}(H), B_{+}(H), B_{+}^{-1}(H)$ stand for the sets of all self-adjoint, invertible self-adjoint, positive, and invertible positive operators, respectively.

The first theorem describes the form of all continuous Jordan triple automorphisms of the set $B_{+}^{-1}(H)$.

ThEOREM 1. Let $\phi: B_{+}^{-1}(H) \rightarrow B_{+}^{-1}(H)$ be a continuous bijective map which satisfies

$$
\phi(A B A)=\phi(A) \phi(B) \phi(A) \quad\left(A, B \in B_{+}^{-1}(H)\right) .
$$

If $H$ is infinite-dimensional, then $\phi$ is either of the form

$$
\phi(A)=U A U^{*} \quad\left(A \in B_{+}^{-1}(H)\right)
$$

or of the form

$$
\phi(A)=U A^{-1} U^{*} \quad\left(A \in B_{+}^{-1}(H)\right)
$$

where $U$ is a unitary or antiunitary operator on $H$.

If $3 \leq \operatorname{dim} H<\infty$, then $\phi$ is either of the form

$$
\phi(A)=(\operatorname{det} A)^{c} U A U^{*} \quad\left(A \in B_{+}^{-1}(H)\right)
$$

or of the form

$$
\phi(A)=(\operatorname{det} A)^{c} U A^{-1} U^{*} \quad\left(A \in B_{+}^{-1}(H)\right)
$$

where $U$ is a unitary or antiunitary operator on $H$ and $c$ is a real number such that $c \neq-1 / \operatorname{dim} H$ in (3) and $c \neq 1 / \operatorname{dim} H$ in (4). 
Our next result describes the structure of all continuous Jordan triple automorphisms of $B_{+}(H)$. This is closely related to our former result [13, Theorem 3] which was used in [1] to determine the so-called sequential automorphisms of the Hilbert space effect algebra.

Theorem 2. Let $\phi: B_{+}(H) \rightarrow B_{+}(H)$ be a continuous bijective map which satisfies

$$
\phi(A B A)=\phi(A) \phi(B) \phi(A) \quad\left(A, B \in B_{+}(H)\right) .
$$

Then $\phi$ is of the form

$$
\phi(A)=U A U^{*} \quad\left(A \in B_{+}(H)\right)
$$

where $U$ is a unitary or antiunitary operator on $H$.

In our last result we determine the continuous Jordan triple automorphisms of the set of all invertible self-adjoint operators. We recall that the group automorphisms of the general linear group of $B(H)$ (the group of all invertible elements of $B(H)$ ) were described in our paper [17].

Theorem 3. Let $\phi: B_{\mathrm{s}}^{-1}(H) \rightarrow B_{\mathrm{s}}^{-1}(H)$ be a continuous bijective map which satisfies

$$
\phi(A B A)=\phi(A) \phi(B) \phi(A) \quad\left(A, B \in B_{\mathrm{s}}^{-1}(H)\right) .
$$

If $H$ is infinite-dimensional, then there exist a unitary or antiunitary operator $U$ on $H$ and a continuous function $\tau: B_{\mathrm{s}}^{-1}(H) \rightarrow\{-1,1\}$ such that $\phi$ is either of the form

$$
\phi(A)=\tau(A) U A U^{*} \quad\left(A \in B_{\mathrm{s}}^{-1}(H)\right)
$$

or of the form

$$
\phi(A)=\tau(A) U A^{-1} U^{*} \quad\left(A \in B_{\mathrm{s}}^{-1}(H)\right) .
$$

If $3 \leq \operatorname{dim} H<\infty$, then there are a unitary or antiunitary operator $U$ on $H$, a real number $c$ and a continuous function $\tau: B_{\mathrm{s}}^{-1}(H) \rightarrow\{-1,1\}$ such that $\phi$ is either of the form

$$
\phi(A)=\tau(A)|\operatorname{det} A|^{c} U A U^{*} \quad\left(A \in B_{\mathrm{s}}^{-1}(H)\right)
$$

or of the form

$$
\phi(A)=\tau(A)|\operatorname{det} A|^{c} U A^{-1} U^{*} \quad\left(A \in B_{\mathrm{s}}^{-1}(H)\right) .
$$

The real number $c$ satisfies $c \neq-1 / \operatorname{dim} H$ in (8) and $c \neq 1 / \operatorname{dim} H$ in (9).

2. Proofs. We begin with the following lemma.

Lemma. Let $H$ be an infinite-dimensional complex Hilbert space and let $\left.\varphi: B_{+}^{-1}(H) \rightarrow\right] 0, \infty[$ be a continuous Jordan triple functional, i.e.,

$$
\varphi(A B A)=\varphi(A) \varphi(B) \varphi(A) \quad\left(A, B \in B_{+}^{-1}(H)\right) .
$$

Then $\varphi$ is identically 1. 
Proof. We assert that $\varphi$ is multiplicative on commuting operators. To see this, first observe that $\varphi(I)=\varphi(I)^{3}$ so that $\varphi(I)$ is 1 . Hence we obtain $\varphi\left(A^{2}\right)=\varphi(A I A)=\varphi(A)^{2}$. This shows that $\varphi(\sqrt{A})^{2}=\varphi(A)$. Let $A, B \in$ $B_{+}^{-1}(H)$ commute. Then

$$
\varphi(A B)=\varphi(\sqrt{A} B \sqrt{A})=\varphi(\sqrt{A})^{2} \varphi(B)=\varphi(A) \varphi(B) .
$$

It follows that

$$
\lambda \mapsto \varphi(\lambda I)
$$

is a continuous multiplicative function on $] 0, \infty[$. The form of such functions can be easily described. In fact, composing a function $f$ of that kind with exp on the right and with log on the left, one obtains a continuous additive function on the real line. By continuity, this function must be linear and hence it is a constant multiple of the identity. Transforming back to the original function we see that $f$ is a power function. Therefore,

$$
\varphi(\lambda I)=\lambda^{c} \quad(\lambda \in] 0, \infty[)
$$

for some real $c$. We shall prove that $c=0$.

Suppose that $c$ is not zero. Then without loss of generality we can assume that $c=1$. Pick an integer $n \geq 3$ and consider the map

$$
A \mapsto \varphi\left(\begin{array}{cccc}
A & 0 & \ldots & \ldots \\
0 & A & 0 & \ldots \\
\vdots & \vdots & \ddots & \vdots
\end{array}\right)
$$

on the set of all $n \times n$ positive definite matrices. Clearly, this is again a continuous Jordan triple functional. The form of such maps on positive definite matrices has recently been described in [16, Theorem 1]. It follows from that result that the map (10) equals a certain power of the determinant. As $c$ above was assumed to be 1 , we conclude that this power is $1 / n$.

The observation above easily implies the following. For any integer $n \geq 3$ and pairwise orthogonal infinite rank projections $P_{1}, \ldots, P_{n}$ with sum $I$ we have

$$
\varphi\left(\lambda_{1} P_{1}+\cdots+\lambda_{n} P_{n}\right)=\left(\lambda_{1} \cdots \lambda_{n}\right)^{1 / n} .
$$

However, decomposing $P_{1}$ into the sum of two mutually orthogonal projections $P_{11}, P_{12}$ of infinite rank we have, on the one hand,

$$
\varphi\left(\lambda_{1}\left(P_{11}+P_{12}\right)+\lambda_{2} P_{2}+\cdots+\lambda_{n} P_{n}\right)=\left(\lambda_{1} \cdots \lambda_{n}\right)^{1 / n}
$$

and on the other hand,

$$
\varphi\left(\lambda_{1} P_{11}+\lambda_{1} P_{12}+\lambda_{2} P_{2}+\cdots+\lambda_{n} P_{n}\right)=\left(\lambda_{1} \lambda_{1} \lambda_{2} \cdots \lambda_{n}\right)^{1 /(n+1)},
$$

SO

$$
\left(\lambda_{1} \cdots \lambda_{n}\right)^{1 / n}=\left(\lambda_{1} \lambda_{1} \lambda_{2} \cdots \lambda_{n}\right)^{1 /(n+1)} .
$$


Since this must hold for all positive real numbers $\lambda_{1}, \ldots, \lambda_{n}$ we obtain an obvious contradiction.

Therefore, the constant $c$ must be zero. Now, a similar argument yields

$$
\varphi\left(\lambda_{1} P_{1}+\cdots+\lambda_{n} P_{n}\right)=1
$$

for all positive real numbers $\lambda_{1}, \ldots, \lambda_{n}$ and pairwise orthogonal infinite rank projections $P_{1}, \ldots, P_{n}$ with sum $I$. It is easy to see that for any finite rank projection $P$ and positive real number $\lambda$, the operator $\lambda P+P^{\perp}$ can be written as the product of two commuting operators $\lambda_{1} P_{1}+\lambda_{2} P_{2}$ and $\mu_{1} Q_{1}+$ $\mu_{2} Q_{2}$, where $P_{1}, P_{2}$ are mutually orthogonal infinite rank projections with sum $I$, and the same holds for $Q_{1}, Q_{2}$. As $\varphi$ is multiplicative on commuting operators, we obtain

$$
\varphi\left(\lambda P+P^{\perp}\right)=1 .
$$

Considering the product of commuting operators of the form $\lambda P+P^{\perp}$, this easily implies that

$$
\varphi\left(\lambda_{1} P_{1}+\cdots+\lambda_{n} P_{n}\right)=1
$$

for any positive real numbers $\lambda_{1}, \ldots, \lambda_{n}$ and pairwise orthogonal projections $P_{1}, \ldots, P_{n}$ with sum $I$, independently of their ranks. Since, by the spectral theorem, every positive invertible operator is the norm limit of a sequence of operators of the form $\lambda_{1} P_{1}+\cdots+\lambda_{n} P_{n}$, we finally conclude that $\varphi$ is everywhere 1.

Proof of Theorem 1. First observe that $\phi(I)=I$. Indeed, $\phi(I)=\phi(I)^{3}$. Since $\phi(I)$ is a positive invertible operator, we obtain $\phi(I)=I$.

We next prove that $\phi$ preserves commutativity in both directions. To show this, we introduce the following operation which was originally defined for the so-called Hilbert space effects which are the positive operators on $H$ bounded by the identity. So, following [2], for arbitrary positive operators $A, B$ we define

$$
A \circ B=\sqrt{A} B \sqrt{A} .
$$

Since $\phi\left(A^{2}\right)=\phi(A I A)=\phi(A) \phi(I) \phi(A)=\phi(A)^{2}$, it follows that $\phi$ preserves the square root, i.e., $\phi(\sqrt{A})=\sqrt{\phi(A)}$ for every $A \in B_{+}^{-1}(H)$. It is now obvious that $\phi$ also preserves the operation $\circ$, i.e.,

$$
\phi(A \circ B)=\phi(A) \circ \phi(B) \quad\left(A, B \in B_{+}^{-1}(H)\right) .
$$

It follows from [2, Corollary 3] that for any positive operators $A, B$ we have $A \circ B=B \circ A$ if and only $A B=B A$. Hence, using (11) we find that $\phi$ preserves commutativity in both directions. Observe that it also follows from (11) that $\phi$ is multiplicative on commuting elements of $B_{+}^{-1}(H)$.

Now, using the continuous function calculus, define a map $\psi$ on the set $B_{\mathrm{s}}(H)$ of all self-adjoint operators by

$$
\psi(S)=\log \phi\left(e^{S}\right) \quad\left(S \in B_{\mathrm{s}}(H)\right) .
$$


It is easy to check that $\psi$ is a bijective map of $B_{\mathrm{S}}(H)$ which preserves commutativity in both directions. Such transformations of $B_{\mathrm{s}}(H)$ have recently been characterized in [18]. Applying [18, Corollary 2] we find that there exists a unitary or antiunitary operator $U$ on $H$ such that for every $S \in B_{\mathrm{s}}(H)$ there is a real-valued bounded Borel function $g_{S}$ on the spectrum $\sigma(S)$ of $S$ such that

$$
\psi(S)=U g_{S}(S) U^{*}
$$

Going back to $\phi$, it is now obvious that for every $A \in B_{+}^{-1}(H)$ there is a real-valued bounded Borel function $f_{A}$ on $\sigma(A)$ such that

$$
\phi(A)=U f_{A}(A) U^{*} .
$$

Clearly, we may assume without serious loss of generality that $U=I$.

So, for every $A \in B_{+}^{-1}(H)$, the operator $\phi(A)$ is a real-valued bounded Borel function of $A$. Hence for any fixed non-trivial projection $P$ there are functions $h_{P}, h_{P}^{\prime}$ from $] 0, \infty[$ into itself such that

$$
\phi\left(\lambda P+P^{\perp}\right)=h_{P}(\lambda) P+h_{P}^{\prime}(\lambda) P^{\perp} .
$$

By the properties of $\phi$, the functions $h_{P}, h_{P}^{\prime}$ are multiplicative and continuous. It follows that they are power functions, i.e., there are real numbers $\alpha(P)$ and $\alpha^{\prime}(P)$ such that

$$
\phi\left(\lambda P+P^{\perp}\right)=\lambda^{\alpha(P)} P+\lambda^{\alpha^{\prime}(P)} P^{\perp} \quad(\lambda \in] 0, \infty[) .
$$

It is easy to see that such numbers can also be chosen in the case when $P$ is trivial, i.e., when $P$ is either 0 or $I$.

Pick arbitrary non-trivial projections $P, Q$ on $H$ which are orthogonal to each other. We compute, on the one hand,

$$
\phi\left(\lambda(P+Q)+(P+Q)^{\perp}\right)=\lambda^{\alpha(P+Q)}(P+Q)+\lambda^{\alpha^{\prime}(P+Q)}(P+Q)^{\perp},
$$

and on the other hand,

$$
\begin{aligned}
\phi(\lambda(P+Q) & \left.+(P+Q)^{\perp}\right)=\phi\left(\left(\lambda P+P^{\perp}\right)\left(\lambda Q+Q^{\perp}\right)\right) \\
& =\phi\left(\lambda P+P^{\perp}\right) \phi\left(\lambda Q+Q^{\perp}\right) \\
& =\left(\lambda^{\alpha(P)} P+\lambda^{\alpha^{\prime}(P)} P^{\perp}\right)\left(\lambda^{\alpha(Q)} Q+\lambda^{\alpha^{\prime}(Q)} Q^{\perp}\right) \\
& =\lambda^{\alpha(P)+\alpha^{\prime}(Q)} P+\lambda^{\alpha^{\prime}(P)+\alpha(Q)} Q+\lambda^{\alpha^{\prime}(P)+\alpha^{\prime}(Q)}(P+Q)^{\perp} .
\end{aligned}
$$

Hence

$$
\alpha(P)+\alpha^{\prime}(Q)=\alpha^{\prime}(P)+\alpha(Q)
$$

which implies that

$$
\alpha(P)-\alpha^{\prime}(P)=\alpha(Q)-\alpha^{\prime}(Q) .
$$

Using this property, it is not hard to verify that $\alpha(\cdot)-\alpha^{\prime}(\cdot)$ is constant on the set of all non-trivial projections. Let this constant be $k$. Define $m(P)=$ 
$\alpha(P)-k$ for any non-trivial projection $P$. Although we do not need this fact, we note that (14) and (15) imply the orthogonal additivity of $m$.

Suppose now that $H$ is finite-dimensional. Let $P_{1}, \ldots, P_{n}$ be pairwise orthogonal non-zero projections with sum $I$ and let $\lambda_{1}, \ldots, \lambda_{n}$ be positive real numbers. Set $A=\lambda_{1} P_{1}+\cdots+\lambda_{n} P_{n}$. Since $\phi$ is multiplicative on commuting operators, we compute

$$
\begin{aligned}
\phi(A) & =\phi\left(\lambda_{1} P_{1}+\ldots+\lambda_{n} P_{n}\right)=\phi\left(\left(\lambda_{1} P_{1}+P_{1}^{\perp}\right) \cdots\left(\lambda_{n} P_{n}+P_{n}^{\perp}\right)\right) \\
& =\phi\left(\lambda_{1} P_{1}+P_{1}^{\perp}\right) \cdots \phi\left(\lambda_{n} P_{n}+P_{n}^{\perp}\right) \\
& =\left(\lambda_{1}^{\alpha\left(P_{1}\right)} P_{1}+\lambda_{1}^{\alpha^{\prime}\left(P_{1}\right)} P_{1}^{\perp}\right) \cdots\left(\lambda_{n}^{\alpha\left(P_{n}\right)} P_{n}+\lambda_{n}^{\alpha^{\prime}\left(P_{n}\right)} P_{n}^{\perp}\right) \\
& =\left(\lambda_{1}^{m\left(P_{1}\right)+k} P_{1}+\lambda_{1}^{m\left(P_{1}\right)} P_{1}^{\perp}\right) \cdots\left(\lambda_{n}^{m\left(P_{n}\right)+k} P_{n}+\lambda_{n}^{m\left(P_{n}\right)} P_{n}^{\perp}\right) \\
& =\lambda_{1}^{k+m\left(P_{1}\right)} \lambda_{2}^{m\left(P_{2}\right)} \cdots \lambda_{n}^{m\left(P_{n}\right)} P_{1}+\cdots+\lambda_{1}^{m\left(P_{1}\right)} \cdots \lambda_{n}^{k+m\left(P_{n}\right)} P_{n} \\
& =\lambda_{1}^{m\left(P_{1}\right)} \lambda_{2}^{m\left(P_{2}\right)} \cdots \lambda_{n}^{m\left(P_{n}\right)}\left(\lambda_{1}^{k} P_{1}+\cdots+\lambda_{n}^{k} P_{n}\right) .
\end{aligned}
$$

This shows that there is a positive real-valued function $\varphi$ on $B_{+}^{-1}(H)$ such that

$$
\phi(A)=\varphi(A) A^{k} \quad\left(A \in B_{+}^{-1}(H)\right) .
$$

By the multiplicativity property of $\phi$, it follows that for any $A, B \in B_{+}^{-1}(H)$ the operator $(A B A)^{k}$ is a positive scalar multiple of $A^{k} B^{k} A^{k}$. It is not hard to verify that this can be the case only when $k= \pm 1$ (the case $k=0$ is excluded because of the bijectivity of $\phi$ ).

Now, returning back to (16), it is clear that $\varphi$ is a continuous Jordan triple functional. We described the general form of such functionals in [16, Theorem 2]. It follows from that result that there exists a real number $c$ such that

$$
\varphi(A)=(\operatorname{det} A)^{c} \quad\left(A \in B_{+}^{-1}(H)\right) .
$$

This implies that either

$$
\phi(A)=(\operatorname{det} A)^{c} A \quad\left(A \in B_{+}^{-1}(H)\right)
$$

or

$$
\phi(A)=(\operatorname{det} A)^{c} A^{-1} \quad\left(A \in B_{+}^{-1}(H)\right) .
$$

Observe that the facts that $c \neq-1 / \operatorname{dim} H$ in the first case and $c \neq 1 / \operatorname{dim} H$ in the second are simple consequences of the injectivity of $\phi$.

Suppose now that $H$ is infinite-dimensional. Following the argument above which has led to (16) we see that there is a non-zero real number $k$ such that $\phi(A) A^{-k}$ is a scalar operator for every $A \in B_{+}^{-1}(H)$ with finite spectrum. By continuity, the same holds for every $A \in B_{+}^{-1}(H)$. Therefore,

$$
\phi(A)=\varphi(A) A^{k} \quad\left(A \in B_{+}^{-1}(H)\right)
$$


for some positive real-valued function $\varphi$ on $B_{+}^{-1}(H)$. Just as above, using the multiplicativity property of $\phi$ we see that $k$ is necessarily either 1 or -1 and that $\varphi$ is a continuous Jordan triple functional. Applying the Lemma we infer that $\varphi \equiv 1$ and the proof is complete.

Proof of Theorem 2. We first prove that $\phi(I)=I$. In fact, for any $A \in B_{+}(H)$ we have $\phi(A)=\phi(I A I)=\phi(I) \phi(A) \phi(I)$. Choosing $A \in B_{+}(H)$ such that $\phi(A)=I$, we obtain $I=\phi(I)^{2}$, which implies that $\phi(I)=I$.

We next assert that $\phi$ preserves invertible operators in both directions. Indeed, this follows from the easy observation that $A \in B_{+}(H)$ is invertible if and only if there exists $B \in B_{+}(H)$ such that $A B A=I$. Since $\phi$ preserves this property, we obtain the assertion.

The restriction of $\phi$ to $B_{+}^{-1}(H)$ gives a bijective transformation of $B_{+}^{-1}(H)$ which preserves the Jordan triple product. Hence, Theorem 1 can be applied. By continuity, in both finite and infinite dimensions, the second possibility appearing in the formulation of Theorem 1 can be excluded. At the same time, in the infinite-dimensional case $\phi$ is of the form (5), while in the finitedimensional case there is a unitary or antiunitary operator $U$ and a real number $c \geq 0$ such that

$$
\phi(A)=(\operatorname{det} A)^{c} U A U^{*} \quad\left(A \in B_{+}(H)\right) .
$$

Since $\phi$ is injective also on the set of singular elements of $B_{+}(H)$, it follows that $c=0$ and the proof is complete.

Proof of Theorem 3. We assert that $\phi(I)$ is either $I$ or $-I$. From $\phi(I)=$ $\phi(I)^{3}$ we deduce that $\phi(I)^{2}=I$. Next, multiplying the equality $\phi(A)=$ $\phi(I A I)=\phi(I) \phi(A) \phi(I)$ by $\phi(I)$ on the left we obtain $\phi(I) \phi(A)=\phi(A) \phi(I)$. Therefore, $\phi(I)$ commutes with every invertible self-adjoint operator. This easily implies that $\phi(I)$ commutes with every bounded linear operator, which implies that $\phi(I)$ is a scalar operator. This proves the assertion.

Without loss of generality we can assume that $\phi(I)=I$. Since $\phi\left(A^{2}\right)=$ $\phi(A I A)=\phi(A) \phi(I) \phi(A)=\phi(A)^{2}$, it follows that $\phi$ preserves invertible positive operators. Thus, Theorem 1 can be applied. In the infinite-dimensional case there is a unitary or antiunitary operator $U$ on $H$ such that $\phi$ is of the form $\phi(A)=U A U^{*}$ or $\phi(A)=U A^{-1} U^{*}$ on $B_{+}^{-1}(H)$. Applying suitable transformations if necessary, we can assume that $\phi$ is the identity on $B_{+}^{-1}(H)$. Let $A \in B_{\mathrm{s}}^{-1}(H)$ and pick an arbitrary $T \in B_{+}^{-1}(H)$. As $A T A \in B_{+}^{-1}(H)$, we compute

$$
A T A=\phi(A T A)=\phi(A) \phi(T) \phi(A)=\phi(A) T \phi(A) .
$$

Since this holds for every $T \in B_{+}^{-1}(H)$, it follows easily that also $A T A=$ $\phi(A) T \phi(A)$ for every $T \in B(H)$. It is easy to see that this implies that $\phi(A)= \pm A$. Of course, the sign here depends on $A$, but due to the continuity 
of $\phi$ this dependence must be continuous as well. This completes the proof in the infinite-dimensional case.

In the finite-dimensional case the argument is practically the same, with an appeal to the finite-dimensional part of Theorem 1.

REMARK. Let us examine the function $\tau$ appearing in Theorem 3. As it is continuous and discrete valued, it must be constant on the components of $B_{\mathrm{s}}^{-1}(H)$. One can prove the non-trivial fact that these components can be characterized in the following way. The invertible self-adjoint operators $A, B$ with spectral measures $E_{A}, E_{B}$ belong to the same component of $B_{\mathrm{s}}^{-1}(H)$ if and only if the spectral projections $E_{A}(] 0, \infty[), E_{B}(] 0, \infty[)$ are equivalent. In the finite-dimensional case this implies that there are exactly $\operatorname{dim} H+1$ such components and each component can be labelled by the common number of positive eigenvalues (counted with multiplicity) of its elements.

3. Remarks. We conclude the paper with some remarks. First of all we note that the forms of our transformations are not only sufficient but also necessary. In fact, this is fairly easy to check in the case of our first two theorems. As for the third one, it follows from the non-trivial fact that $A B A$ and $B$ belong to the same component of $B_{\mathrm{s}}^{-1}(H)$ for all $A, B \in B_{\mathrm{s}}^{-1}(H)$.

Next we remark that using a direct approach to Theorem 2 (not invoking Theorem 1) one could prove that the statement also remains true when $H$ is non-separable or $\operatorname{dim} H=2$. We believe that the same is true for Theorems 1 and 3. However, having no proof, we leave it as an open problem.

Finally, we suspect that the continuity assumption can be omitted in Theorems 1 and 3 if the underlying space is infinite-dimensional (as for Theorem 2, the other approach to its proof mentioned above shows that this assumption is indeed redundant). Of course, in Theorem 3 the assertion on the continuity of $\tau$ should be deleted in that case. Just as above, we do not have the proof, so we leave it as an open problem as well.

\section{References}

[1] S. Gudder and R. Greechie, Sequential products on effect algebras, Rep. Math. Phys. 49 (2002), 87-111.

[2] S. Gudder and G. Nagy, Sequentially independent effects, Proc. Amer. Math. Soc. 130 (2002), 1125-1130.

[3] J. Hakeda, Additivity of *-semigroup isomorphisms among ${ }^{*}$-algebras, Bull. London Math. Soc. 18 (1986), 51-56.

[4] -, Additivity of Jordan *-maps on AW*-algebras, Proc. Amer. Math. Soc. 96 (1986), 413-420.

[5] J. Hakeda and K. Saitô, Additivity of Jordan ${ }^{*}$-maps between operator algebras, J. Math. Soc. Japan 38 (1986), 403-408. 
[6] P. Li and W. Jing, Jordan elementary maps on rings, Linear Algebra Appl. 382 (2004), 237-245.

[7] Z. Ling and F. Lu, Jordan maps of nest algebras, ibid. 387 (2004), 361-368.

[8] F. Lu, Multiplicative mappings of operator algebras, ibid. 347 (2002), 283-291.

[9] - Additivity of Jordan maps on standard operator algebras, ibid. 357 (2002), 121131.

[10] —, Jordan maps on associative algebras, Comm. Algebra 31 (2003), 2273-2286.

[11] —, Jordan triple maps, Linear Algebra Appl. 375 (2003), 311-317.

[12] W. S. Martindale III, When are multiplicative mappings additive?, Proc. Amer. Math. Soc. 21 (1969), 695-698.

[13] L. Molnár, On some automorphisms of the set of effects on Hilbert space, Lett. Math. Phys. 51 (2000), 37-45.

[14] -, On isomorphisms of standard operator algebras, Studia Math. 142 (2000), 295302.

[15] —, Jordan maps on standard operator algebras, in: Functional Equations - Results and Advances, Z. Daróczy and Zs. Páles (eds.), Adv. Math. 3, Kluwer, Dordrecht, 2001, 305-320.

[16] - , A remark to the Kochen-Specker theorem and some characterizations of the determinant on sets of Hermitian matrices, Proc. Amer. Math. Soc., to appear.

[17] L. Molnár and P. Šemrl, Local automorphisms of the unitary group and the general linear group on a Hilbert space, Expo. Math. 18 (2000), 231-238.

[18] —, 一, Non-linear commutativity preserving maps on self-adjoint operators, Quart. J. Math., to appear.

[19] P. Šemrl, Isomorphisms of standard operator algebras, Proc. Amer. Math. Soc. 123 (1995), 1851-1855.

Institute of Mathematics

University of Debrecen

P.O. Box 12

H-4010 Debrecen, Hungary

E-mail: molnarl@math.klte.hu

Received June 20, 2005

Revised version October 14, 2005 